\title{
Hepatitis B virus upregulates the expression of kinesin family member $4 \mathrm{~A}$
}

\author{
CHENG-LIANG ZHU ${ }^{1}$, DUO-ZHI CHENG ${ }^{2}$, FANG LIU $^{3}$, XIAO-HONG YAN ${ }^{3}$, \\ KAI-LANG WU ${ }^{3}$, FU-BING WANG ${ }^{4}$ and XING-HUI LIU ${ }^{5}$ \\ ${ }^{1}$ Department of Clinical Laboratory, Renmin Hospital of Wuhan University, Wuhan, Hubei 430060; \\ ${ }^{2}$ Department of Clinical Laboratory, Taihe Hospital of Hubei Medical College, Shiyan, Hubei 442000; \\ ${ }^{3}$ The State Key Laboratory of Virology, College of Life Sciences, Wuhan University, Wuhan, Hubei 430072; \\ ${ }^{4}$ Department of Clinical Laboratory, Zhongnan Hospital of Wuhan University, Wuhan, Hubei 430071; \\ ${ }^{5}$ Department of Clinical Laboratory, Gongli Hospital, Second Military Medicine University, \\ Shanghai 200135, P.R. China
}

Received July 12, 2014; Accepted April 16, 2015

DOI: $10.3892 / \mathrm{mmr} .2015 .3792$

\begin{abstract}
Hepatitis B virus (HBV) infection is one of the major causes of hepatocellular carcinoma (HCC). Kinesin family member 4A (KIF4A) is a microtubule-based motor protein, which is upregulated in cervical and lung cancer. However, the expression of KIF4A in HBV-associated HCC, and the effect of $\mathrm{HBV}$ on the expression of KIF4A remain to be elucidated. In the present study, the expression profiles of KIF4A were examined in cancerous tissues and paracancerous tissues from patients with HCC, who presented with histories of chronic HBV infection, and the role of HBV in the induction of the expression of KIF4A was investigated. HepG2 cells were transfected with the pHBV1.3, HBV infectious clone and a construct, which contained the luciferase gene under the control of the KIF4A gene promoter. The results demonstrated that the expression of KIF4A was significantly higher in the $\mathrm{HCC}$ tissues than in the paracancerous tissues. HBV activated the KIF4A gene promoter and upregulated the mRNA and protein expression of KIF4A. Furthermore, activation of the gene expression of KIF4A increased in a pHBV1.3 concentration-dependent manner. These results provide novel insights into the understanding of HCC oncogenesis caused by HBV.
\end{abstract}

Correspondence to: Mrs. Fang Liu, The State Key Laboratory of Virology, College of Life Sciences, Wuhan University, 299 Bayi Road, Wuhan, Hubei 430072, P.R. China

E-mail: liufung@126.com

Dr Xing-Hui Liu, Department of Clinical Laboratory, Gongli Hospital, Second Military Medicine University, 219 Miaopu Road, Pudong New Area, Shanghai 200135, P.R. China

E-mail: syliuxh@163.com

Key words: hepatitis B virus, kinesin family member 4A, luciferase activity

\section{Introduction}

Primary hepatocellular carcinoma (HCC), which occurs in the liver parenchymal cells or the epithelial cells of the intrahepatic bile duct, is one of the most clinically common and damaging types of malignant cancer. The high rate of malignancy that is associated with HCC is associated with reduced survival rates in patients (1). In particular, the 5-year survival rate for HCC is $\sim 3-5 \%$, which corresponds to the third highest mortality rate of all types of cancer worldwide $(2,3)$. HCC is primarily caused by hepatitis B virus (HBV) infection. Previous in-depth and extensive investigations of HBV-induced HCC have been performed to identify the specific pathogenic mechanisms by which HBV can cause HCC; however, these mechanisms remain to be fully elucidated (4-6). In our preliminary study, microarray screening was used to identify genes with differences in expression between cancer tissues and paracancerous tissues in patients with $\mathrm{HCC}$ and histories of chronic HBV infection (7). From this screen, kinesin family member 4A (KIF4A) was found to be highly expressed in the tumor tissues relative to the paracancerous tissues. The KIF4A gene maps to Xq13.1 in the human genome and encodes a $140-\mathrm{kDa}$ protein, which is composed of 1,232 amino acids (8-10). KIF4A, which is located predominantly in the cytoplasm and nuclei of cells, is a motor protein that has been closely associated with the intracellular movement of organelles, mitosis and meiosis, the growth and development of tissues and organs, neuronal development and signal transduction (11-13). The present study aimed to evaluate the regulatory effects of HBV on the expression of KIF4A and to examine the molecular mechanisms underlying these effects.

\section{Materials and methods}

Materials. Samples of paracancerous and cancerous tissue were obtained from three male patients $(45,59$ and 64 years old) with HCC and histories of chronic HBV infection, who underwent surgery in Zhongnan Hospital at Wuhan University 
(Wuhan, China). The HepG2 human liver cancer cell line was provided by the China Center for Type Culture Collection at Wuhan University. The pKIF4A-Luc plasmid, containing the luciferase gene driven by the KIF4A gene promoter, and the pHBV1.3 plasmid, which produces an infectious clone of HBV, were constructed in The State Key Laboratory of Virology, College of Life Sciences, Wuhan University (Wuhan, China). TRIzol, a RNA extraction reagent and Lipofectamine 2000, a transfection reagent, were purchased from Invitrogen Life Technologies (Carlsbad, CA, USA). M-MLV reverse transcriptase $(200 \mathrm{U} / \mu \mathrm{l})$ and luciferin, a luciferase substrate, were purchased from Promega Corporation (Madison, WI, USA). A luminometer was purchased from Bio-Rad Laboratories, Inc. (TD-20/20; Hercules, CA, USA). The polyclonal rabbit anti-human anti-KIF4A antibody (1:600; ABU010) was purchased from Sigma-Aldrich (St. Louis, CA, USA), and the horseradish peroxide (HRP)-conjugated goat anti-rabbit secondary antibody (1:5,000; ab6721) was purchased from Abcam (Cambridge, UK). The study was approved by the Ethics Committee of Renmin Hospital of Wuhan University (Wuhan, China) and informed consent was obtained from the patients.

Cell culture and transfection. The HepG2 cells were cultured in RPMI-1640 medium (Gibco Life Technologies, Carlsbad, CA, USA) containing 10\% fetal bovine serum (Invitrogen Life Technologies) and two antibiotics (100 U/ml penicillin and $100 \mu \mathrm{g} / \mathrm{ml}$ streptomycin; Gibco Life Technologies) in an incubator at $37^{\circ} \mathrm{C}$ and $5 \% \mathrm{CO}_{2}$. HepG2 cells were also co-transfected with an empty vector, pBlue ks and pKIF4A Luc. The transfection solutions were created by diluting $0.6 \mu \mathrm{g}$ pHBV1.3 or pBlue ks and $0.2 \mu \mathrm{g}$ pKIF4A Luc and $2 \mu \mathrm{l}$ Lipofectamine 2000 transfection reagent (Invitrogen Life Technologies) into $50 \mu \mathrm{l}$ serum and antibiotic free RPMI-1640 (Gibco Life Technologies) culture medium. Following incubation of these solutions at room temperature for $20 \mathrm{~min}$, the appropriate transfection solutions were transferred to the 24-well plates, and the cells were placed in a $5 \% \mathrm{CO}_{2}$ incubator for continued culture. Prior to transfection, the HepG2 cells were seeded onto 24 - (density, $\sim 1 \times 10^{5}$ cells/well) or 6 -well (density, $\sim 5 \times 10^{5}$ cells/well) plates. Cell transfection was performed as follows: Transfection solutions were created by diluting $0.8 \mu \mathrm{g}$ plasmid DNA and $2 \mu \mathrm{l}$ Lipofectamine 2000 transfection reagent into $50 \mu \mathrm{l}$ serum- and antibiotic-free RPMI-1640 culture medium (24-well plate), or by diluting $4 \mu \mathrm{g}$ plasmid DNA and $6 \mu \mathrm{l}$ Lipofectamine 2000 transfection reagent into $100 \mu \mathrm{l}$ serum- and antibiotic-free RPMI-1640 culture medium (6-well plate). Following incubation of these solutions at room temperature for $20 \mathrm{~min}$, the appropriate transfection solutions were transferred to the cell culture plates, and the cells were placed in a $5 \% \mathrm{CO}_{2}$ incubator for continued culture.

Luciferase assay. The HepG2 cells were cultured for $48 \mathrm{~h}$ at $37^{\circ} \mathrm{C}$ after transfection. The culture medium was then removed, and the cells were washed with phosphate-buffered saline (PBS; O'BioLab, Beijing, China). Lysis buffer (Beijing BLKW Biotech Co., Ltd., Beijing, China) was added to lyse the cells (100 $\mu \mathrm{l} /$ well). Following complete lysis of the cells, $50 \mu \mathrm{l}$ of the cell lysate was mixed with $50 \mu \mathrm{l}$ luciferin and a luminometer was used for determination of the optical density. Each experiment was repeated three times.

Reverse transcription-quantitative polymerase chain reaction $(R T-q P C R)$. The total RNA was first extracted from the tissue samples or the HepG2 cells using TRIzol, followed by treatment with DNase I (5 U/ $\mu$; ; Shanghai Haoran Biotechnology Co., Ltd., Shanghai, China). Subsequently, reverse transcriptase from the GoldScript One-Step RT-PCR kit (Invitrogen Life Technologies) was used to synthesize the complementary DNA (cDNA) using $1 \mu \mathrm{g}$ of each RNA sample. Using the synthesized cDNA as a template, qPCR amplification was performed using the GeneAmp 9700 PCR System (Applied Biosystems Life Technologies, Foster City, CA, USA) and the following primers from Jingmei Biotech Co., Ltd. (Shenzhen, China), which were designed to detect the KIF4A gene: Forward 5'-TCAAGCAGAAACTGACCCTC-3' and reverse 5'-CGTTCAACAGTGCCCAAG-3'. Based on standard amplification procedures, the PCR cycling conditions involved 25 cycles of $94^{\circ} \mathrm{C}$ for $45 \mathrm{sec}, 56^{\circ} \mathrm{C}$ for $45 \mathrm{sec}$ and $72^{\circ} \mathrm{C}$ for 45 sec. $\beta$-actin was used as an internal control, and $5 \mu \mathrm{l}$ each PCR product was analyzed using $1 \%$ agarose gel (Jingmei Biotech Co., Ltd.) electrophoresis.

Western blot analysis. The tissues and HepG2 cells were lysed with $1 \mathrm{X}$ lysis buffer, sonicated on ice with the Ultrasonic Instrument (1 sec/ml; 5 times at setting 5; Shanghai Hao Chong Instrument Co., Ltd., Shanghai, China) and centrifuged at $13,000 \mathrm{xg}$ for $5 \mathrm{~min}$ at $4^{\circ} \mathrm{C}$. The concentrations of protein in the sample supernatants were quantified using the Coomassie Brilliant Blue G-250 (Beinuo Biotech Co., Ltd., Shanghai, China) method. For each western blot, $30 \mu \mathrm{g}$ protein from each sample was mixed with an equal volume of $5 \mathrm{X}$ loading buffer $(0.5 \mathrm{~mol} / 1$ Tris $\cdot \mathrm{HCl}, 2.5 \mathrm{ml}$; DTT, $0.39 \mathrm{~g}$; SDS, $0.5 \mathrm{~g}$; bromophenol blue, $0.025 \mathrm{~g}$; glycerol, $2.5 \mathrm{ml}$ ), boiled for $5 \mathrm{~min}$ in a $100^{\circ} \mathrm{C}$ water bath, and separated by sodium dodecyl sulfate-polyacrylamide gel electrophoresis on a $12 \%$ gel. Following electrophoresis, the proteins were transferred onto a nitrocellulose membrane (Sigma-Aldrich). This membrane was blocked for $2 \mathrm{~h}$ at room temperature in PBS Tween-20 (PBST; O'BioLab) containing 5\% nonfat milk, incubated with 1:600 polyclonal KIF4A antibody for $2 \mathrm{~h}$ at room temperature, washed three times with PBST, incubated with 1:5,000 HRP-conjugated goat anti-rabbit secondary antibody for $1 \mathrm{~h}$, and then washed four times with PBST. An enhanced chemiluminescence system (Biomart, Beijing, China) was used to examine the chromogenic signal from the membrane.

Statistical analysis. The SPSS 13.0 software package (SPSS, Inc., Chicago, IL, USA) was used to process and analyze the experimental data. The data are presented as the mean \pm standard deviation, and differences between the groups were compared using Student's t-test. $\mathrm{P}<0.05$ was considered to indicate a statistically significant difference.

\section{Results}

Elevated expression levels of KIF4A in cancer tissues of patients with $H C C$. In our previous study, DNA microarrays were used to screen for differentially expressed genes in the paracancerous 
and cancerous tissues of patients with $\mathrm{HCC}$, who presented with histories of chronic HBV infection (7). This screen revealed increased expression levels of KIF4A in the cancerous tissues, compared with the paracancerous tissues. To further confirm these results, the present study analyzed paracancerous and cancerous tissue samples from three patients with HCC using RT-qPCR and western blotting, to determine the differences in the mRNA and protein expression levels of KIF4A, respectively. The results of the RT-qPCR and western blot analysis revealed almost no expression of KIF4A in the paracancerous tissues, however significantly higher expression levels of KIF4A were observed in cancerous tissues (Figs. 1 and 2).

Activation of the KIF4A gene promoter by $H B V$. To investigate the effect of HBV on the expression of KIF4A, the HepG2 cells were co-transfected with a plasmid encoding the pHBV1.3 HBV infectious clone and pKIF4A-Luc, in which the luciferase gene is driven by the KIF4A gene promoter. In addition, HepG2 cells were also transfected with an empty vector, pBlue-ks, and were included as a control group. The activities of luciferase in each group were subsequently measured to determine the activation of the KIF4A gene promoter by pHBV1.3. The results demonstrated that transfection with the pBlue-ks empty vector produced $157.5 \pm 12.4 \mathrm{RLU} / \mu \mathrm{g}$ protein of luciferase activity, whereas transfection with the pHBV1.3 vector produced $694.6 \pm 36.5 \mathrm{RLU} / \mu \mathrm{g}$ protein of luciferase activity (Fig. 3). Statistical analysis confirmed the statistical significance of this difference $(\mathrm{P}<0.001)$, indicating that $\mathrm{HBV}$ activated the KIF4A gene promoter in the HepG2 cells.

Different concentrations of pHBV1.3 or pBlue-ks $(0$, $0.2,0.4,0.6$ or $0.8 \mu \mathrm{g} / \mathrm{ml}$ ) were co-transfected with the pKIF4A-Luc construct to determine the concentration dependence of the expression of KIF4A on pHBV1.3 in the HepG2 cells. The luciferase activity experiments demonstrated that luciferase activity increased as the concentration of pHBV1.3 increased. In particular, at pHBV1.3 concentrations of $0,0.2$, $0.4,0.6$ and $0.8 \mu \mathrm{g} / \mathrm{ml}$, luciferase activities of $126.8 \pm 13.4$, $219.8 \pm 16.7,387.6 \pm 21.5,586.5 \pm 228.9$ and $657.6 \pm 35.5 \mathrm{RLU} / \mu \mathrm{g}$ protein were observed, respectively. By contrast, transfection with different concentrations of empty vector produced little change in luciferase activity, as pBlue-ks concentrations of $0.2,0.4,0.6$ and $0.8 \mu \mathrm{g} / \mathrm{ml}$ resulted in luciferase activities of $123.6 \pm 13.8,131.8 \pm 14.6,129.7 \pm 13.5,135.3 \pm 13.4$ and $127.1 \pm 12.7 \mathrm{RLU} / \mu \mathrm{g}$ protein, respectively. Statistical analysis demonstrated that transfection with pHBV1.3 produced significantly higher levels of luciferase activity, compared with transfection with the empty vector $(\mathrm{P}<0.001)$, and that activation of the KIF4A gene promoter by HBV was dose-dependent (Fig. 4).

Upregulation of the mRNA expression of KIF4A by HBV. To investigate the effect of $\mathrm{HBV}$ on the mRNA expression of KIF4A, the HepG2 cells were transfected with different concentrations of pHBV1.3, an infectious clone of HBV. RT-qPCR was used to detect changes in the mRNA expression levels of KIF4A $48 \mathrm{~h}$ after transfection. The results indicated that HBV upregulated the mRNA expression of KIF4A, and that the mRNA expression of KIF4A increased as the concentration of pHBV1.3 increased (Fig. 5). These findings were consistent with those of the luciferase activity analysis, described above.
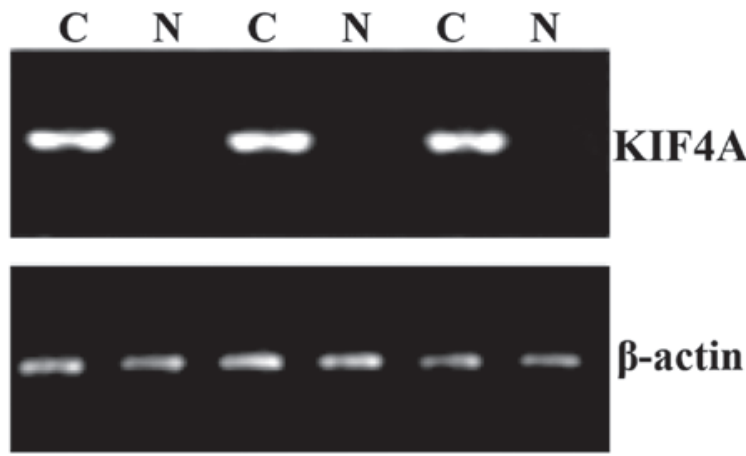

Figure 1. Reverse transcription-quantitative polymerase chain reaction analysis of the mRNA expression of KIF4A in cancerous and paracancerous tissues. C, cancerous tissue; N, paracancerous tissue; KIF4A, kinesin family member $4 \mathrm{~A}$.

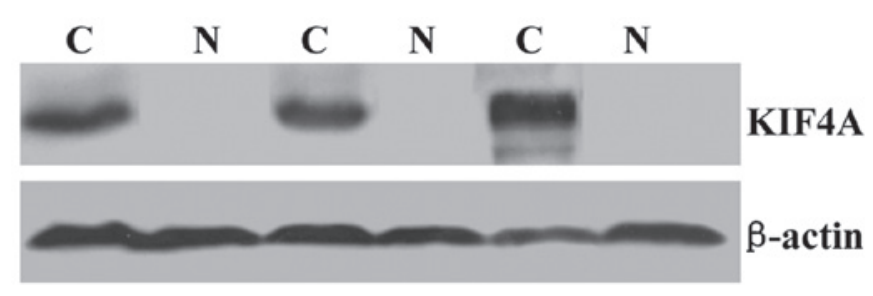

Figure 2. Western blot analysis of the protein expression of KIF4A in cancerous and paracancerous tissues. C, cancerous tissue; N, paracancerous tissue; KIF4A, kinesin family member 4A.

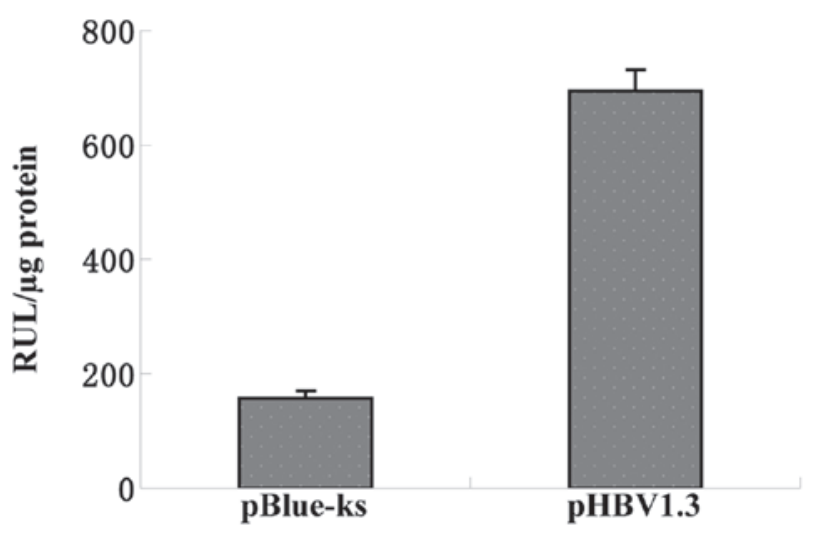

Figure 3. Analysis of the luciferase activity of activation of the kinesin family member 4A gene promoter by HBV. Data are presented as the mean \pm standard deviation. Activity was compared with cells transfected with the pBlue-ks empty vector. HBV, hepatitis B virus.

Upregulation of the protein expression of KIF4A by $H B V$. To examine the effects of $\mathrm{HBV}$ on the protein expression of KIF4A, different concentrations of pHBV1.3 were used to transfect the HepG2 cells. Western blot anaylsis was used to measure the protein expression of KIF4A $48 \mathrm{~h}$ after transfection. The results revealed that $\mathrm{HBV}$ increased the protein expression of KIF4A in the HepG2 cells in a dose-dependent manner (Fig. 6).

\section{Discussion}

$\mathrm{HBV}$ infection is recognized as one of the major causes of primary HCC. Globally, 60-80\% of primary HCC cases 


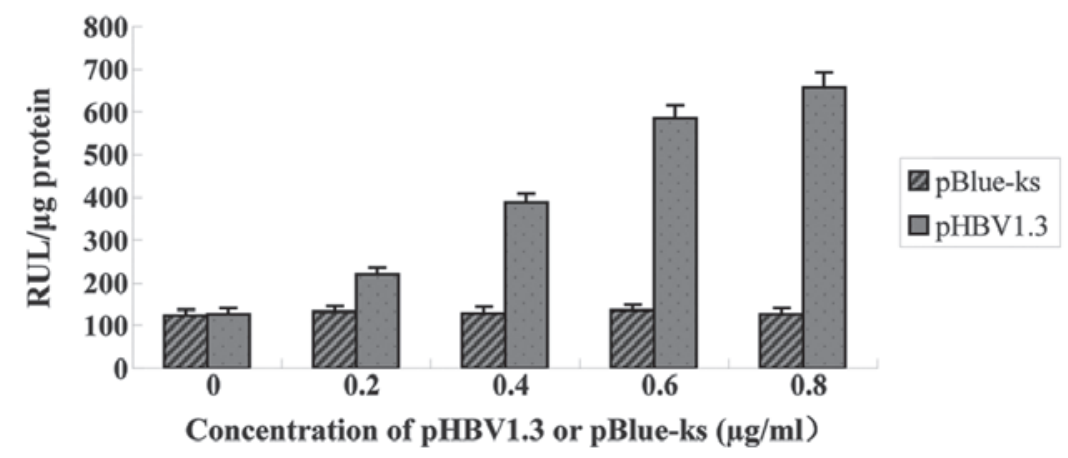

Figure 4. Analysis of the luciferase activity of the activation of the kinesin family member 4A gene promoter by different concentrations of pHBV1.3. Data are presented as the mean \pm standard deviation. Activity was compared with cells transfected with the pBlue-ks empty vector. HBV, hepatitis B virus.

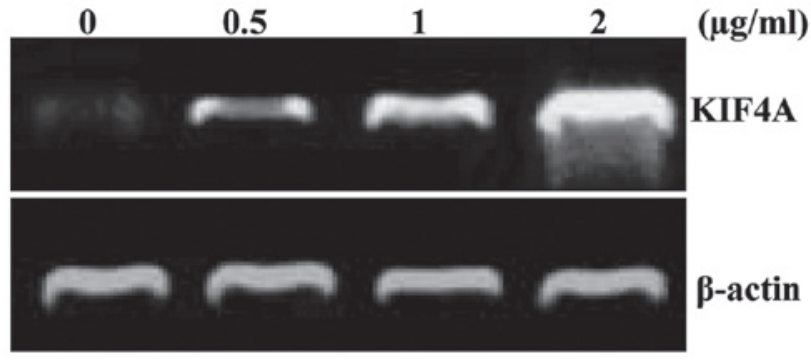

Figure 5. Reverse transcription-quantitative polymerase chain reaction analysis of the effects of transfection with different concentrations of pHBV1.3 on the mRNA expression of KIF4A. HBV, hepatitis B viruis; KIF4A, kinesin family member $4 \mathrm{~A}$.

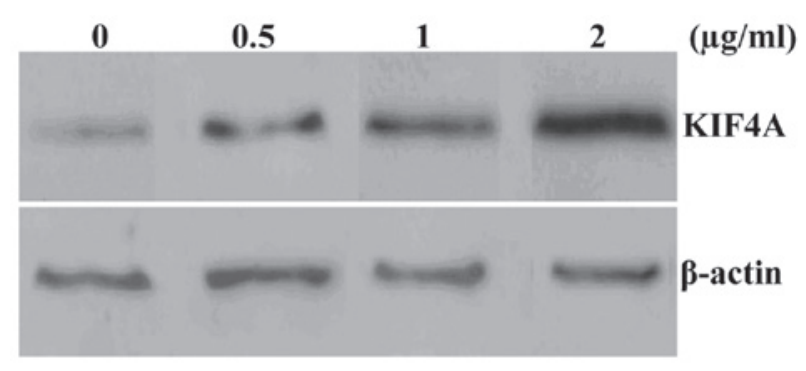

Figure 6. Western blot analysis of the effects of transfection with different concentrations of pHBV1.3 on the protein expression of KIF4A. HBV, hepatitis B viruis; KIF4A, kinesin family member 4A.

are caused by HBV, and $\sim 0.4-0.6 \%$ of patients with chronic HBV infections are diagnosed with HCC each year $(4,5,14)$. To examine the pathogenic and carcinogenic mechanisms of HBV, our prelimanry study used cDNA microarrays to screen for genes, which were expressed at different levels in the cancerous and paracancerous tissues of patients with HCC. The results of this examination indicated that KIF4A was expressed at significantly higher levels in cancerous tissues than in paracancerous tissues.

As DNA microarrays can produce false positive results, the present study obtained cancerous and paracancerous tissue samples from three patients with HCC and histories of chronic HBV infection to confirm the KIF4A-associated results. RT-qPCR and western blotting were used to examine the expression of KIF4A, and the results indicated that KIF4A was expressed at significantly higher levels in the cancerous tissues, comapared with the paracancerous tissue. These findings were consistent with the microarray data.

Following transfection into the HepG2 cells, pHBV1.3, an infectious clone of $\mathrm{HBV}$, can induce the packaging of mature viral particles $(15,16)$. To further examine the molecular mechanisms underlying the upregulation of KIF4A by HBV, the present study constructed pKIF4A-Luc, which contained a luciferase reporter gene driven by the KIF4A gene promoter. The HepG2 cells were co-transfected with pKIF4A-Luc and pHBV1.3, and luciferase reporter assays were performed to determine the role of HBV in regulating the KIF4A gene promoter. Notable, these assays used luciferin as the substrate, which is oxidized in a luciferase-catalyzed reaction to produce bioluminescence that can be quantified using a luminometer (17). The regulatory effects were evaluated based on the levels of luciferase activity following the addition of different quantities of pHBV1.3. The results demonstrated that pHBV1.3 activated the KIF4A gene promoter in a dose-dependent manner. The RT-qPCR and western blotting results revealed that HBV induced the expression of KIF4A in a dose-dependent manner at the mRNA and protein levels, respectively. These findings were consistent with the luciferase activity results.

KIF4A is a multifunctional protein. Previous studies have suggested that KIF4A is involved in responses to DNA damage and DNA repair pathways (12). In particular, KIF4A can interact with the BRCA2 breast cancer susceptibility gene to regulate the BRCA2/Rad51 pathway, which is involved in the response to DNA damage (18). Thus, KIF4A may be important in the incidence and development of breast cancer. KIF4A is overexpressed in cervical and lung cancer, downregulated in gastric cancer, and treatment of non-small cell lung carcinoma cells with specific siRNA to knockdown the expression of KIF4A results in suppression of cancer cell growth $(11,19,20)$.

In the present study, elevated expression levels of KIF4A were observed in tumor tissues from primary tumors of patients with HCC, who presented with histories of chronic HBV infection. A possible mechanism for this observation is that, upon $\mathrm{HBV}$ infection, the gene expression of KIF4A is upregulated in liver tissue, which contributes to liver carcinogenesis. However, the way in which HBV-dependent regulation of KIF4A is associated with liver tumor occurrence requires further investigation.

In conclusion, the present study examined the regulation of KIF4A by HBV at the molecular level and presented preliminary evidence for at least one of the potential carcinogenic mechanisms of HBV. 


\section{Acknowledgements}

This study was supported by research grants from the Major State Basic Research Development Program (973 Program; grant. no. 2012CB518900), the National Clinical Key Subject (grant. no. 2010305), the National Science Foundation of China (grant. no. 81101485 to Dr Cheng-Liang Zhu and no. 31270206 to Dr Kai-Lang Wu), the Open Research Program of the State Key Laboratory of Virology of China (grant. nos. 2011009, 2012007 and 2013004) and the China Postdoctoral Foundation (grant. no. 201104485).

\section{References}

1. Gomaa AI, Khan SA, Toledano MB, Waked I and Taylor-Robinson SD: Hepatocellular carcinoma: Epidemiology, risk factors and pathogenesis. World J Gastroenterol 14 4300-4308, 2008

2. Lim SG, Mohammed R, Yuen MF and Kao JH: Prevention of hepatocellular carcinoma in hepatitis B virus infection. J Gastroenterol Hepatol 24: 1352-1357, 2009.

3. Kim BK, Han KH and Ahn SH: Prevention of hepatocellular carcinoma in patients with chronic hepatitis B virus infection. Oncology 81 (Suppl 1): 41-49, 2011.

4. Nguyen VT, Law MG and Dore GJ: Hepatitis B-related hepatocellular carcinoma: epidemiological characteristics and disease burden. J Viral Hepat 16: 453-463, 2009.

5. Su CH, Lin Y and Cai L: Genetic factors, viral infection, other factors and liver cancer: an update on current progress. Asian Pac J Cancer Prev 14: 4953-4960, 2013.

6. Cougot D, Neuveut C and Buendia MA: HBV induced carcinogenesis. J Clin Virol 34 (Suppl 1): 75-78, 2005.

7. Zhang R, Cao Y, Bai L, et al: The collagen triple helix repeat containing 1 facilitates hepatitis B virus-associated hepatocellular carcinoma progression by regulating multiple cellular factors and signal cascades. Mol Carcinog: Sep 27, 2014 (Epub ahead of print)
8. Martinez NW, Xue X, Berro RG, Kreitzer G and Resh MD: Kinesin KIF4 regulates intracellular trafficking and stability of the human immunodeficiency virus type $1 \mathrm{Gag}$ polyprotein. J Virol 82: 9937-9950, 2008.

9. Wu G and Chen PL: Structural requirements of chromokinesin Kif4A for its proper function in mitosis. Biochem Biophys Res Commun 372: 454-458, 2008.

10. Ha MJ, Yoon J, Moon E, Lee YM, Kim HJ and Kim W: Assignment of the kinesin family member 4 genes (KIF4A and KIF4B) to human chromosome bands Xq13.1 and 5q33.1 by in situ hybridization. Cytogenet Cell Genet 88: 41-42, 2000.

11. Taniwaki M, Takano A, Ishikawa N, et al: Activation of KIF4A as a prognostic biomarker and therapeutic target for lung cancer. Clin Cancer Res 13: 6624-6631, 2007.

12. Wu G, Zhou L, Khidr L, et al: A novel role of the chromokinesin Kif4A in DNA damage response. Cell Cycle 7: 2013-2020, 2008.

13. Nunes Bastos R, Gandhi SR, Baron RD, Gruneberg U, Nigg EA and Barr FA: Aurora B suppresses microtubule dynamics and limits central spindle size by locally activating KIF4A. J Cell Biol 202: 605-621, 2013.

14. Tan YJ: Hepatitis B virus infection and the risk of hepatocellular carcinoma. World J Gastroenterol 17: 4853-4857, 2011.

15. Zhu C, Zhang R, Liu L, et al: Hepatitis B virus enhances interleukin-27 expression both in vivo and in vitro. Clin Immunol 131: 92-97, 2009.

16. Li Y, Xie J, Xu X, et al: Inducible interleukin 32 (IL-32) exerts extensive antiviral function via selective stimulation of interferon lambda1 (IFN-lambda1). J Biol Chem 288: 20927-20941, 2013.

17. Lu L, Wei L, Peng G, et al: NS3 protein of hepatitis C virus regulates cyclooxygenase- 2 expression through multiple signaling pathways. Virology 371: 61-70, 2008.

18. Lee YM and Kim W: Association of human kinesin superfamily protein member 4 with BRCA2-associated factor 35. Biochem J 374: 497-503, 2003.

19. Gao J, Sai N, Wang C, et al: Overexpression of chromokinesin KIF4 inhibits proliferation of human gastric carcinoma cells both in vitro and in vivo. Tumour Biol 32: 53-61, 2011.

20. Narayan G, Bourdon V, Chaganti S, et al: Gene dosage alterations revealed by cDNA microarray analysis in cervical cancer: identification of candidate amplified and overexpressed genes. Genes Chromosomes Cancer 46: 373-384, 2007. 